\title{
Influence of Anisotropy on Dendritic Growth in Binary Alloy with Phase-Field Simulation
}

\author{
Rong Zhen XIAO, Zhi Ping WANG, Chang Sheng ZHU, Wen Sheng LI and Li FENG \\ Key Laboratory of Non-ferrous Metal Alloys and Processing, the Ministry of Education, Lanzhou University of Technology, \\ Lanzhou, 730050, China. E-mail: xrz540909@yeah.net
}

(Received on December 26, 2008; accepted on March 10, 2009)

\begin{abstract}
Some computational results on dendritic growth in binary alloy are obtained by using a phase-field model coupled the solute gradient term. The effect of crystalline anisotropy on the morphological formation, tip steady state and the solute partition is investigated for different dendrites. The interface formation and tip steady state are affected evidently with increase in anisotropy for $\langle 100\rangle$ dendrite growth, but the solute partition coefficient is not significantly influenced. For $\langle 110\rangle$ preferred growth directions, when the anisotropy strength is lower than the critical value, the tip velocity of [110] direction is lower than [101] and [011] directions. As the anisotropy strength crosses the critical value, the tip velocity of [110] direction increases suddenly, larger than the tip velocity of [101] and [011] directions, showing strong solute trapping.
\end{abstract}

KEY WORDS: phase-field model; dendritic growth; fourfold symmetric anisotropy; sixfold symmetric anisotropy; solute distribution.

\section{Introduction}

Microstructure formation is at the center of materials science and engineering, and it is the strategic link between materials processing and behavior. It is very important and realistic to control the microstructure formation of microstructures effectively. The free growth of a single dendrite into an undercooled melt is one of the most fundamental problems in solidification science. In addition to a great deal of experimental research, several theoretical models describing the crystal growth into an undercooled melt have been proposed. ${ }^{1-6)}$

Since the phase-field models (PFM) had been advanced, they have rapidly become very popular for the simulation of microstructures evolution during solidification process. ${ }^{7-9)}$ In early simulation, the PFM was only used to simulate the equiaxed dendrite growth during solidification of pure substances in a supercooled melt. ${ }^{7)}$ The first model was proposed by Wheeler et al. ${ }^{8,9)}$ for alloy solidification, and it was widely called WBM (Wheeler, Boettinger, Mcfadden) model. The WBM model has been extensively used in ideal dilute solutions of binary alloys. ${ }^{10-12)}$ Moreover, a new phase-field model named KKS model had been advanced by Kim et al. ${ }^{13,14)}$ then this model is equivalent with the WBM model, but has a different definition of the free energy density for the interfacial region.

The strength of the crystalline anisotropy crucially influence the interface pattern formation and the primary branches of an equiaxed grain emerges along with $\langle 100\rangle$ directions in cubic crystals. Karma and Rappel ${ }^{15)}$ simulated three-dimensional equiaxed dendrites with $\langle 100\rangle$ growth directions for large enough anisotropy using phase-field method. But when the preferred growth directions are $\langle 110\rangle$ instead of $\langle 110\rangle$, there will be six primary branches in two-dimensional space. In the present work, a phase-field model cooperated solute concentration gradient corrections is developed for simulating the dendritic growth of $\mathrm{Ni}-\mathrm{Cu}$ alloy, and the pattern formations of $\langle 100\rangle$ dendrites and $\langle 110\rangle$ dendrites are simulated. The effect of the strength of the crystalline anisotropy on the morphological formation, growth velocity and the solute distribution is investigated for different dendrites.

\section{Numerical Model}

\subsection{Controlled Equation}

In the phase-field model, a new variable, the phase-field $\phi(x, y, t)$ is introduced to indicate the physical state of the system at each point, $\phi$ takes on 0 in solid and 1 in liquid, and changes between 1-0 deeply but smoothly over a thin transition layer that plays the role of the classical sharp interface. The present model is directly based on the formation given by Warren and Boettinger ${ }^{10)}$ for isothermal approximation solidification. According to Landau-Ginzburg theory, the system entropy can be postulated the form as

$$
S=\int\left(s(\phi, e, c)-\frac{\varepsilon^{2}}{2}|\nabla \phi|^{2}-\frac{\delta^{2}}{2}|\nabla c|^{2}\right) d x^{3}
$$

where integration is performed over the system volume; $s$ is the thermodynamic entropy density, $e$ is the internal energy density, and the coefficients $\varepsilon$ and $\delta$ describe the extent of the phase field and the solute field gradient term corrections. In this paper, the diffuse of the solute is considered 
stable, so the value of $\delta$ is constant. $c$ is the molar fraction of a solute $B$ in solvent $A$; and in this paper, $A$ is $\mathrm{Ni}, B$ is $\mathrm{Cu}$. Here, we require that $s(0, e, c)$ and $s(1, e, c)$ are the classical thermodynamic entropy densities for homogeneous solid and liquid. We ignore the volume changes, which should be a good approximation for the systems, thus gaining the phase-field model as

$$
\begin{array}{r}
\frac{\partial \phi}{\partial t}=M_{\phi}\left\{\nabla \cdot \varepsilon^{2} \nabla \phi-\left[(1-c) H^{A}+c H^{B}\right]\right\} \\
\frac{\partial c}{\partial t}=\nabla \cdot D_{c}\left[\nabla c+\frac{v_{m}}{R} c(1-c)\left(H^{B}-H^{A}\right) \nabla \phi\right. \\
\left.-\frac{v_{m}}{R} c(1-c) \nabla\left(\delta^{2} \nabla^{2} c\right)\right]
\end{array}
$$

where $v_{m}$ is the molar volume, $R$ is the gas constant, and $\varepsilon$ represents anisotropy of the interfacial energy. It can be written as

$$
\varepsilon(\theta)=\bar{\varepsilon}\left[1+\gamma \cos k\left(\theta-\theta_{0}\right)\right] .
$$

where $\gamma$ is the intensity of anisotropy in the surface energy, $k$ specifies the mode number, $\theta$ is an angle between the interface normal and the orientation of the crystal lattice, and the approximation expression is $\theta=\arctan \left(\phi_{y} / \phi_{x}\right)$. In the present work, $\theta_{0}$ is a constant.

In Eqs. (2), (3), $H^{A}$ can be defined as

$$
H^{A}=W^{A} g^{\prime}(\phi)+30 g(\phi) L^{A}\left(\frac{1}{T}-\frac{1}{T_{m}^{A}}\right)
$$

$H^{B}$ has the same expression as $H^{A} . M_{\phi}$ is a phase-field parameter related to the interface dynamic, defined as

$$
M_{\phi}=(1-c) M^{A}+c M^{B}
$$

In Eq. (3), $D_{c}$ is the solute diffusivity and defined as

$$
D_{c}=D_{s}+p(\phi)\left(D_{l}-D_{s}\right)
$$

where $D_{s}$ and $D_{l}$ are the diffusivities in the liquid and solid, respectively. In Eq. (5), the $g(\phi)$ is defined as

$$
g(\phi)=\phi^{2}(1-\phi)^{2}
$$

which is a symmetric double well potential with equal minima at $\phi=0$ and $\phi=1$.

With the choice $p(\phi)=\phi^{3}\left(10-15 \phi+6 \phi^{2}\right)$, the reasons for these particular choices are elucidated that the bulk solid and liquid are described by $\phi=0$ and $\phi=1$, for every value of temperature respectively.

We can obtain the relationship of the phase-field parameters $M^{A, B}, W^{A, B}, \bar{\varepsilon}$ and the material properties by solving the diffusion equations ${ }^{16)}$

$$
\begin{aligned}
& M^{A}=\frac{\left(T_{m}^{A}\right)^{2} \beta^{A}}{6 \sqrt{2} L^{A} \xi^{A}} \\
& W^{A}=\frac{3 \sigma^{A}}{\sqrt{2} T_{m}^{A} \xi^{A}} .
\end{aligned}
$$

$$
\bar{\varepsilon}^{2}=\frac{6 \sqrt{2} \sigma^{A} \xi^{A}}{T_{m}^{A}}=\frac{6 \sqrt{2} \sigma^{B} \xi^{B}}{T_{m}^{B}}
$$

$M^{B}$ and $W^{B}$ have the same expression as the $M^{A}$ and $W^{A}$. Where $T_{m}^{A}$ and $T_{m}^{B}$ are the melting point of pure $A$ and $B$ respectively, $\beta$ is the interface dynamic coefficient, $L^{A}$ is the heat latent of pure $A, \xi$ is the interface thickness, and $\sigma$ is the interface energy.

\subsection{Thermal Noise}

In this paper, the stochastic thermal noise is imposed to the solid/liquid interface to simulate the evolution process of the solid/liquid interfacial pattern formation, and the noise can be introduced by

$$
\frac{\partial \phi}{\partial t} \rightarrow \frac{\partial \phi}{\partial t}-M_{\phi} \alpha r \operatorname{lgg}(\phi)\left[(1-c) H_{A}+c H_{B}\right] \ldots
$$

where $\alpha$ is the magnitude of the fluctuation, $r$ is a random number between +1 and -1 . Here, $\alpha=0.4$.

\subsection{Initial Conditions}

In present work, a square computational domain of size $1200 \times 1200$ grids is used in the simulations. The ZeroNeumann boundary condition is imposed to the $c$ and $\phi$ at the boundaries in the bulk region. For simplifying the computation, we shall consider a purely isothermal model for this work. The energy conservation equation will be ignored and a constant temperature field will be imposed. ${ }^{16)}$ Therefore, the initial conditions can be required as

$$
\begin{aligned}
& \text { When } \quad x^{2}+y^{2} \leq r_{0}^{2}, \phi=0, T=T_{0}, c=c_{s}^{e q} \ldots \\
& \text { When } \quad x^{2}+y^{2}>r_{0}^{2}, \phi=1, T=T_{0}, c=c_{\infty} \ldots
\end{aligned}
$$

where $r_{0}$ is the radius of an initial nucleus, $x$ and $y$ are the coordinate axes, $T_{0}$ is the initial temperature of the undercooled melt, $T_{0}=1574 \mathrm{~K}$ and the initial undercooling $\Delta T=20.5 \mathrm{~K}$, yielding the equilibrium interface concentration of $c_{s}^{e q}=0.3994$ and $c_{\infty}=0.40831$.

In order to accord with the convergent condition, the step spacing $\Delta y=\Delta x=2.41 \times 10^{-6} \mathrm{~cm}$ and the time step $\Delta t=$ $1.1 \times 10^{-8} \mathrm{~s}$. They are given by

$$
\Delta t<\frac{\Delta x^{2}}{4 D_{l}}
$$

In the simulations, simple centered finite differencing formula can be used throughout the domain for the spatial derivatives using uniform grid. The material properties are appropriate to nickel-copper, and this system exhibits a nearly ideal solution behavior. ${ }^{10)}$ We take the material parameters as Table 1.

\section{Results and Discussion}

\subsection{Fourfold Symmetric Growth}

In usual phase-field approaches, the anisotropy of the solidification interface energy $\varepsilon(\theta)$, is simply assumed to have fourfold symmetric anisotropy as ${ }^{9-17,23-27)}$ 
Table 1. The material properties of the $\mathrm{Ni}-\mathrm{Cu}$ alloy.

\begin{tabular}{lcc}
\hline Parameter & $\operatorname{Nickel}(A)$ & $\operatorname{Copper}(B)$ \\
\hline$T_{m}(\mathrm{~K})$ & 1728 & 1358 \\
$L\left(\mathrm{~J} / \mathrm{cm}^{3}\right)$ & 2350 & 1728 \\
$v_{m}\left(\mathrm{~cm}^{3} / \mathrm{mole}\right)$ & 7.42 & 7.42 \\
$\sigma\left(\mathrm{J} / \mathrm{cm}^{2}\right)$ & $3.7 \times 10^{-5}$ & $2.9 \times 10^{-5}$ \\
$\beta\left(\mathrm{cm}^{2} / \mathrm{Ks}\right)$ & 0.33 & 0.39 \\
$D_{s}\left(\mathrm{~cm}^{2} / \mathrm{s}\right)$ & $1.0 \times 10^{-9}$ & $1.0 \times 10^{-9}$ \\
$D_{l}\left(\mathrm{~cm}^{2} / \mathrm{s}\right)$ & $1.0 \times 10^{-5}$ & $1.0 \times 10^{-5}$ \\
$\delta(\mathrm{mole} / \mathrm{cm})$ & $2.0 \times 10^{-6}$ & $2.0 \times 10^{-6}$ \\
\hline
\end{tabular}

$$
\varepsilon(\theta)=\bar{\varepsilon}\left[1+\gamma_{1} \cos 4\left(\theta-\frac{\pi}{2}\right)\right]
$$

where $\gamma_{1}$ is the intensity of fourfold symmetric crystalline anisotropy. In this case, dendrite trunks and arms grow along well-defined crystallographic directions, which are the $\langle 100\rangle$ growth directions. There will be four growth orientations of a crystal seed in the two dimensional $x y$-plane, as described in Fig. 1.

Figure 1 shows the pattern formations of the solute distribution and phase-field profiles for a nickel-copper alloy with different anisotropy coefficients, and the growth time $t=1.221,1.001,0.539 \mathrm{~ms}$ for Fig. 1(a)-1(c) respectively. It can be seen that, the tendency of the side-branches to begin their growth in a direction is not perpendicular to the primary stalk, but later develop a growth axis that is perpendicular to the primary stalk, and the necking behavior is seen to be significantly on the root of the side arms, as described by Warren and Boettinger. ${ }^{10)}$ However, some side-branches have undergone obvious remelting during competitive growth in this calculation. At the same time, the solute ahead of interface in undercooled melt enriches. The maximum value of the solute concentration is corresponding to the regions of the dendrites between the secondary arms, and the solute concentration in the primary arm's spines is relatively low, as well as in the secondary arm's spines. But an increased level of anisotropic strength is known to increase the severity of micro-segregation, which is shown nicely in Fig. 1(b) and 1(c) (the black line denoting contours of the lowest solute concentration in the solid). We also can find that, the secondary arms develop well and the arm spacing becomes narrow with the increasing of the anisotropy strength. But the tendency of the side-branches to begin their growth in a direction is perpendicular to the primary stalk at high anisotropy of $\gamma_{1}=0.070$, and the dendrites become slender with the increment of anisotropy. As proved by Eggleston et al., ${ }^{27)}$ missing orientations occur for $\gamma_{1} \geq 1 / 15\left(\gamma_{1} \approx 0.066\right)$, and the facet interface appears in this region. Figure 2 shows the dendritic tip formation for $\gamma_{1}=0.070$. As can be seen, the interface varies discontinuously and the equilibrium shape becomes unstable with edges and corners due to the presence of missing orientations.
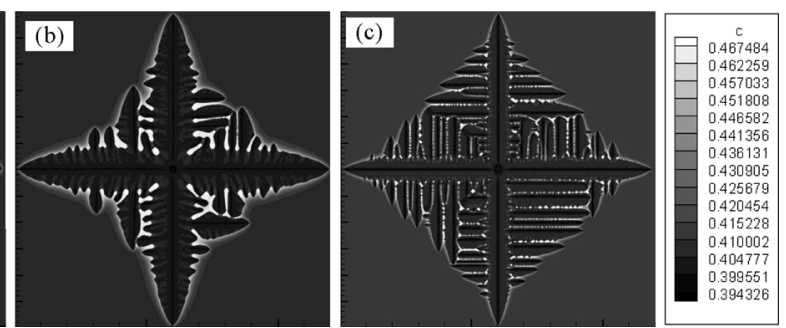

lots of the concentration and phase field profiles with fourfold symmetric anisotro(a) $\gamma=0.035$, (b) $\gamma=0.055$, (c) $\gamma=0.07$

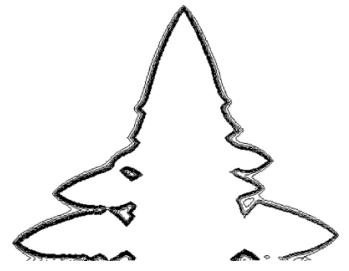

Fig. 2. The dendritic tip formation with $\gamma=0.07$.

\subsection{Sixfold Symmetric Growth}

When the solidification conditions become more favorable to $\langle 110\rangle$ growth morphologies, the dendrites will have six preferable prism orientations in the two dimensional projective plane of (111) coherent twin plane, and a dendrite with hexagonal lattice symmetry of the dominant hexagonal close-packed phase may be obtained.

As pointed out in Ref. 17), the earlier problem of the dendritic growth of a nickel-copper alloy under large enough anisotropy with $\langle 110\rangle$ preferred growth directions in three dimensions was computed by Henry. Moreover, Zhao and Heinrich examined the crystal growth with hexagonal lattice symmetry. ${ }^{18,19)}$

The dendritic growth along $\langle 110\rangle$ crystallographic directions instead of $\langle 100\rangle$ crystallographic directions is supposed to be due to a change in the anisotropy of the liquid/solid surface energy and surface stiffness. ${ }^{20)}$ In this case, the anisotropy $\varepsilon(\theta)$ is specified by the following anisotropic model with sixfold symmetry

$$
\varepsilon(\theta)=\bar{\varepsilon}\left[1+\gamma_{2} \cos 6\left(\theta-\frac{\pi}{3}\right)\right]
$$

where $\gamma_{2}$ is the intensity of sixfold symmetric crystalline anisotropy. In this case, the neighbor dendrite trunks incline to one another at an angle of $60^{\circ}$, as presented in Fig. 3 .

It can be found that the anisotropy has significant effects on the dendritic pattern formation and the tip operating behavior. The dendrite morphology is the "snow-shape crystal" when the hexagonal anisotropy function has to be integrated into the model, as shown in Fig. 3. The calculation time for each result is $1.93 \mathrm{~ms}, 1.32 \mathrm{~ms}, 1.01 \mathrm{~ms}$ and $0.66 \mathrm{~ms}$. The first feature of note is that the side-branches increase with the increment of the anisotropy. And at the beginning the side-branches grow in a direction is perpendicular to the parent branches, but later develop a growth axis that is parallel to the neighborly primary stalk. In order to quantify $\gamma_{2}$, a number of simulations have been performed and a critical value of the intensity of sixfold anisotropy strength has been obtained, $\gamma_{2}=0.02$. When the 

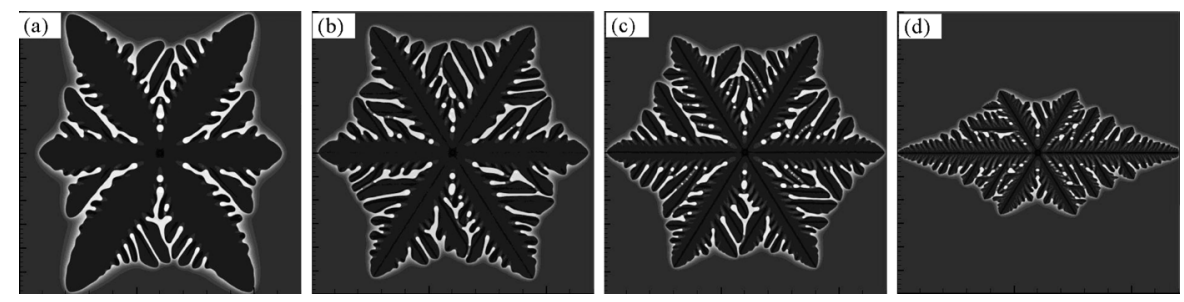

Fig. 3. Plots of the concentration and phase field profiles with hexagonal crystalline anisotropy. (a) $\gamma=0.005$, (b) $\gamma=0.020$, (c) $\gamma=0.025$, (d) $\gamma=0.030$.

intensity of sixfold symmetric crystalline anisotropy is lower than the critical value $\gamma_{2}=0.02$, the main trucks

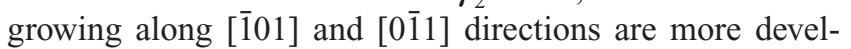
oped than the trucks growing along [110] direction, as shown in Fig. 3(a). As the anisotropy crosses the critical value, [1110] dendritic branch becomes more developed than others. When the intensity of anisotropy strength $\gamma_{2}=0.030$, the growth tendency of other main branches has been strongly restrained, and a typical $\langle 110\rangle$ texture of feathery grain can be observed, as shown in Fig. 3(c and d). At the late stage of the calculation, the coarsening of the secondary arms occurs. An advanced secondary arm produced by [011] dendrite along [1 $1 \overline{0} 0]$ direction grows competitively over its parent branches, and begins to develop a new main branch. The tip growth velocities will be discussed in the next section.

\subsection{Effect of Anisotropy on Tip Operating Behavior}

Over the last 30 years, the significant theoretical and experimental progress has been made in understanding the role of crystalline anisotropy. The effect of anisotropy on the liquid/solid interface pattern formation and the tip operating behavior can be taken into account. ${ }^{20-26)}$ In order to simplify the computation, the average growth rate of driving five grids is considered as the tip velocity in this time. In this simulation, the dendrite tip velocity and tip radius are calculated by

$$
\begin{aligned}
& V_{\text {tip }}=\frac{\partial \varphi / \partial t}{|\nabla \varphi|} \\
& R_{\mathrm{tip}}=\frac{2 \phi_{x} \phi_{y} \phi_{x y}-\phi_{y}^{2} \phi_{x x}-\phi_{x}^{2} \phi_{y y}}{\sqrt{\left(\phi_{x}^{2}+\phi_{y}^{2}\right)^{3}}}
\end{aligned}
$$

Figure 4 shows Variations of steady-state tip velocity and tip radius as a function of anisotropy strength for $\langle 100\rangle$ dendrites. It can be found that with the increase of anisotropy strength, the tip velocity increases accordingly, while the tip radius decreases suddenly. But it is worth to be pointed out Ivantsov theory is only suitable for lower anisotropy strength because of neglecting the impact of anisotropy on tip operating behavior. In particular, as the intensity of anisotropy $\gamma$ crosses the value 0.15 , the phenomenon of the decrease in velocity as the anisotropy crossed the critical value performed by Kim et al. ${ }^{21)}$ has not been confirmed. We are unsure of the general validity of this simulation, but note that Warren and Boettinger ${ }^{10)}$ have proved that a value of $\Delta t=(\Delta x)^{2} / 5 D_{l}$ works well for not too large choices of the anisotropy.

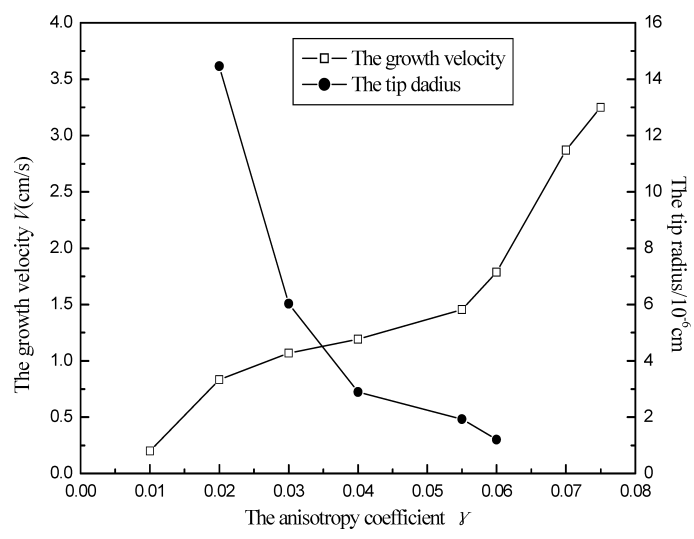

Fig. 4. Variations of steady-state tip velocity and tip radius calculated at several different values of $\gamma$ for $\langle 100\rangle$ directions.

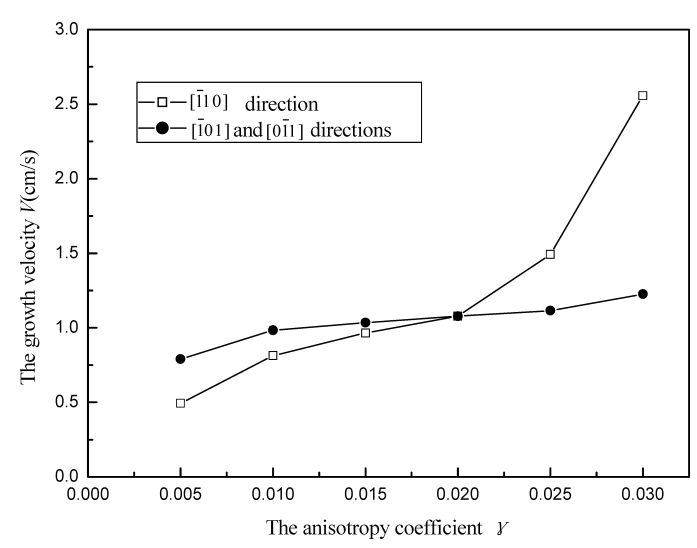

Fig. 5. Variations of tip velocities with different growth orientations calculated at several different values of $\gamma$ for $\langle 110\rangle$ directions.

Figure 5 shows the variations of dendritic tip velocity profiles with different growth orientations calculated at several different values of anisotropy strength for $\langle 110\rangle$ dendrites. As can be seen, the tip velocities of [1110] and [101] directions increase monotonically with anisotropy as expected. But, it should be noted that when the anisotropy strength is lower than the critical value 0.02 , the tip velocity of [1110] direction is lower than the tip velocities of [101] and [011] directions. With further increase in anisotropy, the tip velocities increase, and reach an equal value at anisotropy $\gamma_{2}=0.02$, then the tip velocity of [1 10$]$ direction increases suddenly, larger than the tip velocities of [101] and $[0 \overline{1} 1]$ directions.

\subsection{Effect of Anisotropy on Solute Distribution}

Phase-field simulations have also been conducted to ex- 

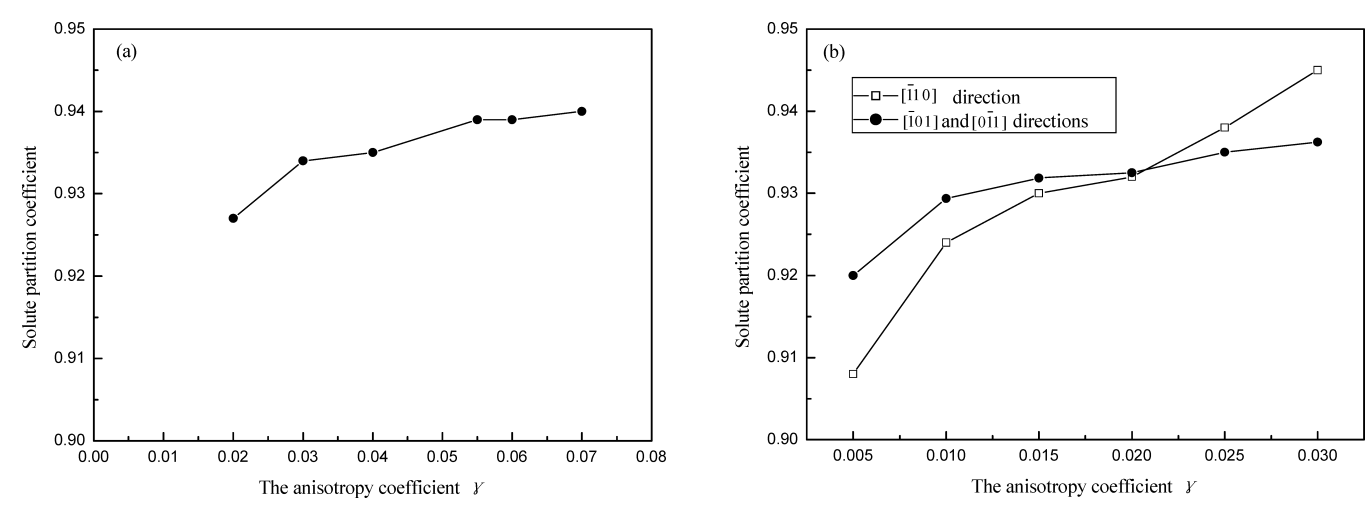

Fig. 6. Solute partition coefficient $v s$. anisotropy for different growth orientations: (a) $\langle 100\rangle$ dendrite, (b) $\langle 110\rangle$ dendrite.

plore the effect of the imposed anisotropy on the solute distribution. A Landau-Ginzburg free-energy function depends on the bulk free-energy density of the system. In present phase-field model, the term of $(\nabla c)^{2}$ is necessary to predict the solute distribution in the calculation region.

Figure 6 shows variations of solute partition coefficient $k$ with different favorable growth directions. We would like to be sure that $k$ is not significantly affected as we increase the anisotropy strength for $\langle 100\rangle$ dendrites, as shown in Fig. 6(a). But for $\langle 110\rangle$ dendrites, as shown in Fig. 6(b), the calculated solute partition coefficients vary significantly with the low increment of the intensity of anisotropy $\gamma$ in [110] direction, and when $\gamma=0.02$, the both solute partition coefficients reach equal value $k=0.932$ for [110] and [101] directions. Because the switch from $\langle 100\rangle$ to $\langle 110\rangle$ growth morphology is attributed to alteration of anisotropy, and the effect of anisotropy on $\langle 110\rangle$ dendritic growth velocity is more available. With the increment of $\gamma$, the noise can be amplified quickly, and then the surface energy cannot restrain effectively the amplification in the finite interface thickness. But the solid diffusivity $\left(\sim 10^{-9} \mathrm{~cm} / \mathrm{s}\right)$ is significantly lower than the growth velocity, so the solute in the solid has no enough time to be redistributed. As a result, the solute trapping is severe.

\section{Conclusion}

A phase-field model of binary alloy solidification is developed by coupled the term of $(\nabla c)^{2}$, bases on the formation given by Warren and Boettinger, the effect of the strength of the crystalline anisotropy on the morphological formation, growth velocity and the solute distribution is investigated for $\langle 100\rangle$ and $\langle 110\rangle$ dendrites.

The steady state of the dendrite tip is influenced by the anisotropy. For $\langle 100\rangle$ dendrite growth, the tip velocity increases and the side-branches are more developed with increase in anisotropy, but the solute partition coefficient is not significantly affected. The tip velocities of [110] and [101] directions increase monotonically with anisotropy for $\langle 110\rangle$ dendrite growth. When the anisotropy strength is lower than the critical value $\gamma=0.02$, the tip velocity of [110] direction is lower than the tip velocities of [101] and [011] directions. As the anisotropy strength crosses the critical value, the tip velocity of [110] direction increases sud-

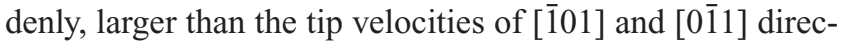
tions, showing strong solute trapping.
Last, it should be noted that it is not clear how to set exactly the value of the anisotropy coefficient and what its connection with real physical parameters is. These simulation results may not be quantitatively correct. However, qualitatively the predicted behavior is in agreement with what is expected at low undercooling.

\section{Acknowledgement}

This work was supported by the National Natural Science Foundation of China (NO. 50804019), and the Open foundation of Key Laboratory of Non-ferrous Metal Alloys and Processing of Ministry of Education (NO. EKL07007) in Lanzhou University of Technology.

\section{REFERENCES}

1) J. Lipton and M. E. Glicksman: Mater. Sci. Eng. A, 65 (1984), 57.

2) M. P. Anderson, D. J. Srolovitz, G. S. Grest and P. S. Sahni: Acta Metall., 32 (1984), 783.

3) J. A. Spittle and S. G. Brown: Acta Metall., 37 (1989), 1803.

4) M. Rappaz and C. A. Gandin: Acta Metall., 41 (1993), 350.

5) C. A. Gandin and M. Rappaz: Acta Metall., 42 (1994), 2233.

6) M. Ode, J. S. Lee, S. G. Kim, W. T. Kim and T. Suzuki: ISIJ Int., 40 (2000), 870.

7) A. A. Wheeler, W. J. Boettinger and G. B. McFadden: Phys. Rev. E, 45 (1992), 7424.

8) A. A. Wheeler, W. J. Boettinger and G. B. McFadden: Phys. Rev. E, 47 (1993), 1893.

9) R. Kobayashi: Physica D, 63 (1993), 410.

10) J. A. Warren and W. J. Boettinger: Acta Metall. Mater., 43 (1995), 689.

11) C. S. Zhu, Z. P. Wang, T. Jing and R. Z. Xiao: Acta Phys. Sin., 55 (2006), 1502

12) C. S. Zhu, Z. P. Wang, T. Jing and R. Z. Xiao: Trans. Nonferrous Met. Soc. China, 16 (2006), 760.

13) S. G. Kim, W. T. Kim and T. Suzuki: Phys. Rev. E, 58 (1998), 3316.

14) S. G. Kim, W. T. Kim and T. Suzuki: Phys. Rev. E, 60 (1999), 7186.

15) A. Karma and W. J. Rappel: Phys. Rev. E, 57 (1998), 4323.

16) I. Loginova, G. Amberg and J. Agern: Acta Mater., 49 (2001), 573.

17) W. J. Boettinger, S. R. Coriell, A. L. Greer, A. Karma, W. Kurz, M. Rappaz and R. Trivedi: Acta Mater, 48 (2000), 43.

18) P. Zhao and J. C. Heinrich: J. Comput. Phys., 173 (2001), 765.

19) P. Zhao and J. C. Heinrich: Int. J. Numer. Meth. Eng., 56 (2003), 1533 .

20) S. Henry, T. Minghetti and M. Rappaz: Acta Mater., 46 (1998), 6431.

21) S. G. Kim and W. T. Kim: J. Cryst. Growth, 275 (2005), 355.

22) C. S. Zhu, B. C. Liu, T. Jing and W. F. Feng: Mater. Trans., 46 (2005), 15.

23) A. M. Mullis: Comput. Mater. Sci., 36 (2006), 345.

24) L. J. Tan and N. Zabaras: J. Comput. Phys., 211 (2006), 36.

25) G. B. Mcfadden, S. R. Coriell and R. F. Sekerka: Acta Mater., 48 (2000), 3177.

26) Y. Suwa, Y. Saito and H. Onodera: Comput. Mater. Sci., 40 (2007), 40.

27) J. J. Eggleston, G. B. Mcfadden and P. W. Voorhees: Physica D, 150 (2001), 91 . 